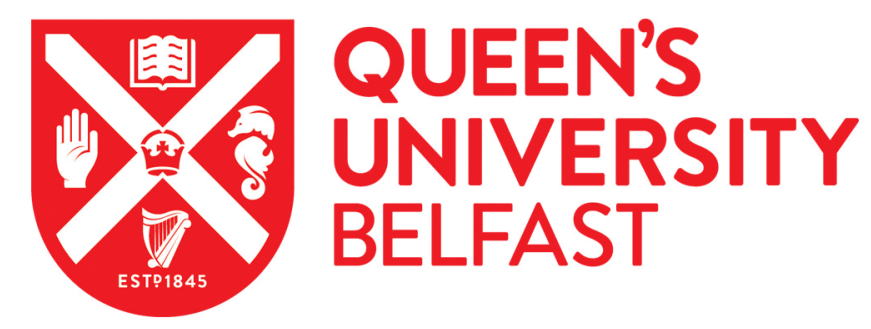

\title{
On the Mechanism of the lodide-Triiodide Exchange Reaction in a Solid-State Ionic Liquid
}

Grossi, J., Kohanoff, J. J., English, N. J., Bringa, E. M., \& Del Pópolo, M. G. (2017). On the Mechanism of the lodide-Triiodide Exchange Reaction in a Solid-State lonic Liquid. Journal of Physical Chemistry B, 121(26), 6436-6441. https://doi.org/10.1021/acs.jpcb.7b01034

Published in:

Journal of Physical Chemistry B

Document Version:

Peer reviewed version

Queen's University Belfast - Research Portal:

Link to publication record in Queen's University Belfast Research Portal

Publisher rights

Copyright 2019 ACM. This work is made available online in accordance with the publisher's policies. Please refer to any applicable terms of use of the publisher.

\section{General rights}

Copyright for the publications made accessible via the Queen's University Belfast Research Portal is retained by the author(s) and / or other copyright owners and it is a condition of accessing these publications that users recognise and abide by the legal requirements associated with these rights.

Take down policy

The Research Portal is Queen's institutional repository that provides access to Queen's research output. Every effort has been made to ensure that content in the Research Portal does not infringe any person's rights, or applicable UK laws. If you discover content in the Research Portal that you believe breaches copyright or violates any law, please contact openaccess@qub.ac.uk. 


\title{
On the Mechanism of the Iodide-Triiodide Exchange Reaction in a Solid- State Ionic Liquid
}

\author{
Joás Grossi, ${ }^{1,2}$ Jorge J. Kohanoff, ${ }^{2,}{ }^{*}$, a) Niall J. English, ${ }^{3}$ Eduardo M. Bringa ${ }^{1}$ and Mario G. Del \\ Pópolo ${ }^{1,2, *, b)}$ \\ ${ }^{1}$ CONICET \& Facultad de Ciencias Exactas y Naturales, Universidad Nacional de Cuyo, \\ Mendoza, Argentina. \\ ${ }^{2}$ Atomistic Simulation Centre, School of Mathematics and Physics, Queen's University \\ Belfast, University Road, Belfast BT7 1NN, Northern Ireland. \\ ${ }^{3}$ School of Chemical and Bioprocess Engineering, University College Dublin, Belfield, \\ Dublin 4, Ireland.
}

\begin{abstract}
Efficient charge transport has been observed in iodide-based room temperature ionic liquids when doped with iodine. In order to investigate preferred pathways for the iodide $\left(\mathrm{I}^{-}\right)$-totriiodide $\left(\mathrm{I}_{3}^{-}\right)$exchange reaction, and to clarify the origin of this high ionic conductivity, we have conducted electronic structure calculations in the crystal state of 1-butyl-3methylimidazolium iodide ([BMIM][I]). Energy barriers for the different stages of the iodineswapping process, including the reorientation of the $\mathrm{I}^{-} \cdots \mathrm{I}_{3}^{-}$moiety, were determined from minimum energy paths as a function of a reaction coordinate. Hirshfeld charges and structural parameters, such as bond lengths and angles, were monitored during the reaction. Several bond-exchange events were observed with energy barriers ranging from 0.17 to $0.48 \mathrm{eV}$ and coinciding with the formation of a twisted $\mathrm{I}^{-} \cdots \mathrm{I}_{3}^{-}$complex. Striking similarities were observed in the mechanics and energetics of this charge-transfer process in relation to solidstate superionic conductors.
\end{abstract}

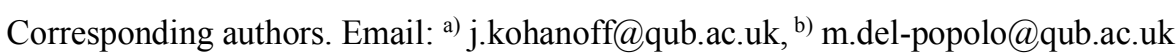




\section{Introduction}

Room-temperature ionic liquids (RTILs) are low-volatility molten salts composed of molecular cations and anions that have, by definition, a melting point below $100^{\circ} \mathrm{C} .{ }^{1}$ They exhibit chemical, rheological and electrical properties that render them versatile alternatives to conventional solvent-based systems. Applications of RTIL range from electrolytes for solar cells ${ }^{2}$, fuel cells, ${ }^{3}$ supercapacitors and batteries, ${ }^{4}$ to lubricants and heat-transfer fluids ${ }^{5}$, and solvents for green synthesis and catalysis. ${ }^{6}$ In particular, high ionic conductivity is an important property, exploited in dye-sensitised solar cells (DSSCs), so that some form of redox electrolyte (usually $\mathrm{I}^{-} / \mathrm{I}_{3}^{-}$in an organic solvent) allows continual replenishment of charge according to the following mechanism: ${ }^{7}$ the electron-hole can transfer from the dye to the electrolyte, while electrons injected into the semiconductor move through it into the external circuit, until these charge carriers recombine at the cathode, closing the circuit. Indeed, being able to extend the DSSC lifecycle almost indefinitely through the replacement of a volatile electrolyte solution with a low-volatility solvent, usually an iodide-based RTIL, offers much promise. ${ }^{8-10}$

The remarkably efficient charge transport - perhaps unexpectedly so when compared to similarly viscous systems - observed upon iodine addition to iodide-based ionic liquids, ${ }^{11-17}$ appears to be based on a bond-exchange process ${ }^{17}$ that in many ways resembles the Grotthuss mechanism for protons diffusion in water. ${ }^{18}$ This mechanism has even been suggested to rationalise the large electrical conductivity of molten polyiodides, ${ }^{19,20}$ although this interpretation is still inconclusive. Wachter et al. have found evidence for such non-Stokesian behaviour in charge transport in binary blends of 1-ethyl-3-methylimidazolium tetrafluoroborate ([EMIM] $\left.\left[\mathrm{BF}_{4}\right]\right)$ and 1-methyl-3-propylimidazolium iodide ([MPIM][I]), ${ }^{21}$ whilst Bentley et al. determined charge transfer in 1-ethyl-3-methylimidazolium bis(trifluoromethanesulphonyl)imide $\left(\left[\mathrm{C}_{2} \mathrm{MIM}\right]\left[\mathrm{NTf}_{2}\right]\right)$ in a manner consistent with a Grotthuss-type bond-exchange reaction. ${ }^{22}$

In any event, establishing the mechanism and energetics of the $\mathrm{I}^{-} / \mathrm{I}_{3}^{-}$charge-transfer process in a solid state RTIL (in which iodine anions couple and swap between $\mathrm{I}^{-}$and $\mathrm{I}_{3}^{-}$in a background provided by the cations lattice) remains an open question, not of impractical significance in the wider context of solid-state RTIL electrolytes for DSSCs. ${ }^{8}$ It is also a computationally feasible first step towards understanding charge transport in disordered, liquid or glassy, iodide-based RTIL. 
Here, using state-of-the-art electronic structure calculations, we elucidate charge-transfer pathways in a representative RTIL crystal, composed of 1-butyl-3-methylimidazolium iodide ([BMIM][I]), with added iodine. In so doing, we establish a transport mechanism which is reminiscent, in terms of energetics, of that observed in solid-state superionic conductors. ${ }^{23}$

\section{Methodology}

A sample of 32 ion pairs of [BMIM][I] was constructed by replicating the unit cell of the crystal in a $2 \times 2 \times 2$ supercell, yielding 832 atoms. An extra (nominally electroneutral) iodine $\mathrm{I}_{2}$ molecule was added adjacent to an iodide $\left(\mathrm{I}^{-}\right)$anion, to form a triiodide ion $\left(\mathrm{I}_{3}^{-}\right)$. This facilitates 'swapping' and the development of $\mathrm{I}^{-} / \mathrm{I}_{3}^{-}$exchange in the crystal state, including the reorientation of the $\mathrm{I}^{-} \cdots \mathrm{I}_{3}^{-}$moiety. The addition of $\mathrm{I}_{2}$ resulted in an electroneutral orthorhombic simulation box containing 834 atoms, subject to periodic boundary conditions (PBC), with lattice parameters equal to $16.561,21.577$ and $23.997 \AA$ corresponding to atmospheric pressure. ${ }^{24}$ Six distinct $\mathrm{I}_{2}$-placement strategies (near a selected $\mathrm{I}^{-}$) were considered with different orientational configurations of the resultant $\mathrm{I}_{3}^{-}$, corresponding to energetically feasible states that would result from $\mathrm{I}^{-} \cdots \mathrm{I}_{3}^{-}$bond-exchanges. These configurations are available in the Supplementary Information. Initial energy-minimisation relaxation on these candidate structures was performed with the molecular-mechanics (MM) forcefield of Canongia Lopes et al. for [BMIM] cations, ${ }^{25}$ whilst iodide ions were treated using a charge of $-1 e$, and Lennard-Jones $12-6$ parameters of $0.07 \mathrm{kcal} / \mathrm{mol}$ and $5.4 \AA$. The charge on the triiodide ion was equally distributed among the three atoms, which thus carried $-\frac{1}{3} e$ each, while Lennard-Jones parameters were the same as those for iodide. The Ewald method was used to handle long-range electrostatic interactions subject to periodic boundary conditions, ${ }^{26}$ and a cut-off of $8 \AA$ was applied to all real-space interactions. MM energyminimisations were performed with the LAMMPS code. It was found that all six structures and $\mathrm{I}^{-} \cdots \mathrm{I}_{3}^{-}$configurations remained stable during minimisation.

Following reassuring molecular-mechanics (MM) findings of structural stability, Density Functional Theory (DFT) calculations under $\mathrm{PBC}$, as implemented in the $\mathrm{CP} 2 \mathrm{~K}^{27}$ package, were used to confirm the stability of these same six systems. The DFT calculations employed the generalised gradient approximation in the $\mathrm{PBE}^{28}$ flavour of the exchange-correlation functional, supplemented with Grimme's D2 dispersion correction. ${ }^{29,30}$ All the electrons, core and valence, were treated explicitly, with the Kohn-Sham orbitals expanded in a $6-31 G^{*}$ basis set,. The electronic density was expanded in plane waves using a 350 Ry cut-off. 
Geometry optimisation was first performed on all six MM-relaxed structures (with supercell volume constant), and little change in overall configuration and $\mathrm{I}^{-} \cdots \mathrm{I}_{3}^{-}$placement was observed, leading to confidence in the consistency of MM and DFT relaxation. To check for pressure-effects and box dilation, we also performed whole-box volume-optimisation for selected configurations imposing a pressure of 1 bar. For the strict [BMIM][I] form without added iodine (consisting of 832 atoms), the volume was $5.47 \%$ smaller than the experimental value, without any substantial change in the configuration. The importance of the dispersion correction can be judged by an overestimation of the volume by $16.7 \%$ when only PBE was used. A similar behaviour of functionals for ionic liquid crystals had already been reported for a family of related systems. ${ }^{31}$ The volume underestimation of the dispersion-corrected PBE should be partially compensated by thermal expansion, since experimental data corresponds to a temperature of $93 \mathrm{~K},{ }^{24}$ while calculations were performed at $\mathrm{T}=0 \mathrm{~K}$. When optimizing the volume with the inclusion of the iodine molecule, the volume increased by less than $2 \%$. In any case, this cell relaxation did not change the configurations appreciably, and left the $\mathrm{I}^{-} \cdots \mathrm{I}_{3}^{-}$arrangements practically intact. Based on this analysis, we are confident that the crystal-phase density is reasonable for the current purposes, and not too different from that of the (viscous) liquid state of [BMIM][I]. ${ }^{32}$

We then evaluated energetic pathways to move, in a concatenated manner, between the six different $\mathrm{I}^{-} \cdots \mathrm{I}_{3}^{-}$bond-exchange states using the Nudged Elastic Band approach. ${ }^{33}$ The paths joining consecutive pairs of states were sampled using tens of intermediate steps, each of which underwent a full geometry optimization. This procedure gave rise to a minimum energy reaction-profile segmented into several windows, which allowed for the location of transition states, as well as for the estimation of energy barriers for each step along the pathway.

\section{Results and Discussion}

Fig. 1 shows, schematically, the $\mathrm{I}^{-} \cdots \mathrm{I}_{3}^{-}$bond-exchange process. Note that the approach of the $\mathrm{I}^{-}(4)$ need not be 'head-on' (i.e., collinear vis-à-vis the 2-3 vector), nor indeed the departure of the leaving $\mathrm{I}^{-}(1)$. In fact, it is generally not so in the condensed phase, especially in the crystalline environment. In order to characterize the exchange process, we defined a reaction coordinate $\xi=d_{21}-d_{43}$, which, in practice, is the difference in bond-length between the breaking (1-2) and the nascent (3-4) I-I chemical bonds. Note that this does not depend on a collinear 'line-up' of all four ions, so it is therefore suitable to capture curvature effects in 
the non-collinear arrangement of the $\mathrm{I}^{-} \cdots \mathrm{I}_{3}^{-}$moiety, that appears almost inevitably in the crystal state. In Fig. S1 (cf. Supplementary Information) we report a gas-phase study of the potential energy surface of the $\mathrm{I}^{-} \cdots \mathrm{I}_{3}^{-}$subsystem as a function of $\xi$, for various $\mathrm{d}_{41}$ separations, via DFT single-point calculations. In this case, we enforce a collinear arrangement, as the 'head-on' approach of 4 and departure of 1 is preferred in vacuo. We see that when $\mathrm{d}_{41}$ is less than, or up to, $\sim 10.5 \AA$, a single energy minimum is evident for $\xi=0$ (i.e., $\mathrm{d}_{43}=\mathrm{d}_{21}$ ), and a symmetric $\mathrm{I}_{4}^{2-}$ complex is formed. However, not unexpectedly, once $\mathrm{d}_{41}$ exceeds $\sim 10.5 \AA$ (of the order of three times the elemental van der Waals diameter of $\sim 3.96$ $\AA^{34}$ ), two minima develop, one for $\mathrm{I}^{-} \cdots \mathrm{I}_{3}^{-}$and other for $\mathrm{I}_{3}^{-} \cdots \mathrm{I}^{-}$, corresponding to the approach of $\mathrm{I}^{-}(4)$ and departure of $\mathrm{I}^{-}(1)$, respectively. Due to electrostatic repulsion, the overall energy decreases as $\mathrm{d}_{41}$ increases, and the distance between $\mathrm{I}^{-}$and $\mathrm{I}_{3}^{-}$grows, such that by $\mathrm{d}_{41}=\sim 11.5 \AA$, the two-state minima are lower in energy than the single-state (equallyspaced) minimum at $\mathrm{d}_{41} \sim 10 \AA$.

Having reviewed the relatively straightforward case of the gas-phase energy landscape, we present energies for traversing the pathways between the six crystal states in Fig. 2. We traverse a full cycle from, and back to state 1 - with energy set at zero for configuration ' $a$ ' on the left. The first activated event corresponds to a reorientation of $\mathrm{I}_{3}^{-}$(first upper panel), with an energy barrier $\Delta E_{1}$ of $0.39 \mathrm{eV}$. The bottom panel, labelled as "Reorientation 1", shows the system's energy as a function of the $\mathrm{I}_{3}^{-}$angular displacement, $\alpha$, measured with respect to its initial orientation (a). As the internal structure of $\mathrm{I}_{3}^{-}$does not change during the reorientation, the energy barrier stems from the reorganization of the crystal lattice. The next step along the pathway is a non-collinear bond exchange (second panel), with a barrier $\Delta E_{3}$ of $0.48 \mathrm{eV}$. Note that the reverse exchange shows a significantly lower barrier, $\Delta E_{4}=0.31 \mathrm{eV}$, due to the anisotropy of the crystal environment. After this bond exchange, a more favourable, energy-relaxing reorientation 2 occurs (third panel), followed by a second and a third exchange event (fourth and fifth panels) with barriers of $\Delta E_{7}=0.26 \mathrm{eV}$ and $\Delta E_{9}=0.17$ $\mathrm{eV}$ for the forward process, and slightly larger values for the reverse exchanges $\left(\Delta E_{8}=0.30\right.$ $\mathrm{eV}$ and $\Delta E_{10}=0.27 \mathrm{eV}$ ). Clearly, the rate-determining step is the first exchange in the somewhat awkward orientational geometry, where $\Delta E_{3}$ is $0.48 \mathrm{eV}$, while the last two, less energetic, exchanges depict more symmetric and collinear transition states.

Given the importance of crystal-state orientations, we studied the angle of departure of $\mathrm{I}^{-}(1), \beta$ (relative to the vector 2-3), in further detail. In Fig. 3, we present the energy as a 
function of $\beta$ along each of the three exchange paths. It is evident that the stable state at the end of the first reorientation, and before the first exchange, has the most 'twisted' and noncollinear arrangement of the four ions, with the highest relative energy amongst the stable configurations, and the highest barrier $\Delta E_{3}$ in Fig. 2 . This is reflected in the largest value of $\beta$ experienced along the first exchange pathway in Fig. 3 (almost $60^{\circ}$ at the transition state, TS). The lower-energy, more geometrically-relaxed exchanges 2 and 3 (cf. Fig. 2) manifest themselves in lower $\beta$ values along the corresponding exchange pathways in Fig. 3 (with respective at-TS $\beta$ maxima of 20 and $31^{\circ}$ ). In other words, steric constraints in the crystalline environment that enforce unnatural orientations of the $\mathrm{I}^{-} \cdots \mathrm{I}_{3}^{-}$moiety, lead to higher energy minima and barriers. It is therefore expected that the less constrained geometries in the liquid phase lead to lower diffusion barriers and higher conductivities.

Consideration of the charge evolution during reorientation and bond exchange is a matter of importance, and is rather instructive and revealing. To this end, we computed Hirshfeld charges $^{35}$ on the iodine ions during the reorientation and bond-exchange pathways (cf. Fig. 4). For reorientations, the central atom in the triiodide atom retains a charge of essentially $-\frac{1}{3} e$. In the first reorientation the outer atoms are almost a 'mirror image' of each other, as they swap electron density during the subsequent 'priming' for bond exchange. The second (downhill) reorientation does not experience any major charge redistributions. Interestingly, the overall charge on the nominally $-1 e$ charged $\mathrm{I}_{3}^{-}$is close to $-1.4 e$. Since elemental iodine is more electronegative than $\mathrm{C}, \mathrm{N}$ or $\mathrm{H}$ atoms in [BMIM], the typical anionic charges on triiodide are somewhat greater than $1 e$ in magnitude due to polarization and charge transfer effects (on the average such effects seem to be slightly smaller on individual iodide anions and [BMIM] cations), with system electroneutrality maintained automatically by PBC-based DFT. ${ }^{27}$ For all three bond-exchange events, similar, not-unexpected trends are seen (cf. Fig. 4): the approaching $\mathrm{I}^{-}$ion gradually reduces its negative charge from (almost) -1 towards around $-0.6 e$ as it becomes incorporated into the $\mathrm{I}_{3}^{-}$moiety, whilst the simultaneouslydeparting iodine anion $\left(\mathrm{I}_{3}^{-} \rightarrow \mathrm{I}^{-}\right)$increases its negative-charge magnitude from that level towards $-1 e$ again, in a 'mirror-image' fashion. In addition, the central two atoms in the $\mathrm{I}^{-} \cdots \mathrm{I}_{3}^{-}$moiety transfer charge between themselves in the same symmetric vein, by and large, during each of the three bond-exchange processes, as they shift 'personality' from the outer ion in contact with the approaching $\mathrm{I}^{-}$ion (with charge of $\sim-0.4$ to $-0.5 e$ ) to becoming the middle triiodide atom (with charge around $-\frac{1}{3} e$ ). 
Taking into consideration the observed behaviour of the mechanistic steps underpinning reorientation and bond exchange, a consistent picture of charge transfer emerges. The ion'hopping' cycle is not unlike the Grotthuss mechanism prevalent in the liquid state, except this typically has rate-limiting energy barriers of $\sim 0.2$ to $0.3 \mathrm{eV} \cdot{ }^{12-17}$ The present ratedetermining barrier $\Delta E_{3}$ of $\sim 0.5 \mathrm{eV}$ (cf. Fig. 2) during the first bond-exchange process is much more similar to those seen in solid-state superionic conductors, e.g., a barrier of $\sim 0.4-$ $0.5 \mathrm{eV}$ for Li-ion diffusivity in sulphide-based solid-state superionic conductors like $\mathrm{Li}_{10} \mathrm{GeP}_{2} \mathrm{~S}_{12}$, and related crystals. ${ }^{23}$ However, we have also found bond-exchange barriers as low as $0.17 \mathrm{eV}$, whenever the [BMIM][I] lattice favours the formation of a relatively linear and relaxed transition state. Moreover, Grotthuss-like steps are not the entire story. In between those, the triiodide has to reposition itself for the next bond-exchange, and this is achieved by means of Stokes-like diffusive steps that exhibit lower barriers, more akin to those observed in liquid/glassy RTIL samples.

\section{Conclusions}

Energetically- and structurally- feasible pathways for $\mathrm{I}^{-} / \mathrm{I}_{3}^{-}$exchange in the crystal state of $[\mathrm{BMIM}][\mathrm{I}]$ have been identified. The energy barriers for each part of the iodine-swapping process, including reorientation of the $\mathrm{I}^{-} \cdots \mathrm{I}_{3}^{-}$moiety, were determined from paths as a function of a reaction coordinate $\xi$, as well as the evolution of Hirshfeld charges. Energy barriers ranging from $0.17 \mathrm{eV}$ to $0.48 \mathrm{eV}$ were observed, which are similar to those of ion diffusion in solid-state superionic conductors. The overall bond-exchange process resembles a Grotthuss-like transport mechanism. In this work we have computed the minimum energy profile following a specific sequence (path) of stable configurations of the triiodide. This path is not unique. There are other possible sequences that will also transfer the $I_{3}^{-}$species across the unit cell, and the possibility that there are other paths with lower barriers is not excluded. Our choice, however, was based on joining the configurations that seemed more amenable to facile $\mathrm{I}_{3}^{-}$transfer.

Clearly, the simulation of this process at finite (perhaps elevated) temperatures would be of interest for a variety of applications, such as solid-state RTIL electrolytes for DSSCs, ${ }^{8}$ and for simulation-led, predictive design of optimal RTIL-based electrolytes. In view of the large energy barriers determined here, biased ab initio molecular-dynamics methods would be required for this. The molecular simulation of liquid-state $\mathrm{I}^{-} / \mathrm{I}_{3}^{-}$exchange is an altogether more challenging prospect: although the energy barriers are likely to be lower, in the general 
$\sim 0.2-0.3 \mathrm{~V}$ region, ${ }^{12-17}$ it would be beneficial to use $\mathrm{QM} / \mathrm{MM}$ or reactive-forcefield approaches to sample properly that panoply of different nuclear configurations in the liquid phase. Such a project remains a goal for the future, in our effort to realise simulation-led design of optimally-conducting electrolytes.

\section{Supporting Information}

Six distinct configurations, each with the $\mathrm{I}_{3}{ }^{-}$molecule adopting a different orientation, used as initial/final state in the five concatenated NEB calculations of Figure 2. We also provide the full set of atomic coordinates for each of the five NEB windows. Details of gas-phase energies of the $\mathrm{I}^{-\cdots} \mathrm{I}_{3}{ }^{-}$moiety as a function of separation, as well as that in the crystal state.

\section{Acknowledgements}

The authors thank Dr. Aaron Byrne for useful discussions. This project has received funding from the European Union's Horizon 2020 research and innovation programme under grant agreement No. 643998. M.G.D.P and E.M.B. acknowledge financial support from CONICET, SECTyP-UNCUYO, and FONCyT (PICT-2015-1835 , PICT-2012-2759 and PICT-2014-0696). We are also grateful for computational support from the UK national high performance computing service, ARCHER, for which access was obtained via the UKCP consortium and funded by EPSRC grant ref. EP/K013564/1. 


\section{References}

1. Ohno, H. Electrochemical Aspects of Ionic Liquids, John Wiley \& Sons 2011

2. Grätzel, M. Photoelectrochemical Cells, Nature 2001, 414, 338 -344.

3. Xu, W.; Angell, C. A. Solvent-Free Electrolytes with Aqueous Solution-Like Conductivities, Science 2003, 302, 422- 425 .

4. Seki, S.; Kobayashi, Y.; Miyashiro, H.; Ohno, Y.; Usami, A.; Mita, Y.; Watanabe, M.; Terada, N. Highly Reversible Lithium Metal Secondary Battery Using a Room Temperature/Mixture and a Surface-coated Cathode Active Material, Chem. Commun. 2006, 544 -545.

5. Ye, C.; Liu, W.; Chen, Y.; Yu, L. Room-Temperature Ionic Liquids: a Novel Versatile Lubricant, Chem. Commun. 2001, $2244-2245$.

6. Wasserscheid, P.; Keim, P. Ionic Liquids in Synthesis, Wiley-VCH 2003.

7. Kawano, R. et al., High performance Dye-Sensitised Solar Cells using Ionic Liquids as their Electrolytes. J. Photochem. Photobiol. A, 2004. 164, 87-92.

8. Yamanaka, N.; Kawano, R.; Kubo, W.; Masaki, N.; Kitamura, T.; Wada, Y.; Watanabe, M.; Yanagida, S. Dye-Sensitized $\mathrm{TiO}_{2}$ Solar Cells Using Imidazolium-Type Ionic Liquid Crystal Systems as Effective Electrolytes, J. Phys. Chem. B 2007, 111, 4763-4769.

9. Han, Y., Pringle, J. M. \& Cheng, Y.-B. Improved Efficiency and Stability of Flexible Dye Sensitised Solar Cells on ITO/PEN Substrates Using an Ionic Liquid Electrolyte. Photochem. Photobiol. 2015. 91, 315-22.

10. Pringle, J.M.; Armel, V. The Influence of Ionic Liquid and Plastic Crystal Electrolytes on the Photovoltaic Characteristics of Dye-sensitized Solar Cells, Inter. Rev. Phys. Chem. 2011, 30, 371 407

11. Kubo, W.; Kitamura, T.; Hanabusa, K.; Wada, Y.; Yanagida, S. Quasi-solid-state-sensitized Solar Cells using Room Temperature Molten Salts and a Low Molecular Weight, Chem. Commun. 2002, $374-375$.

12. Kawano, R.; Watanabe, M. Equilibrium Potentials and Charge Transport of $\mathrm{I}^{-} / \mathrm{I}_{3}^{-}$Redox Couple in an Ionic Liquid, Chem. Commun. 2003, 330-331.

13. Kawano, R.; Watanabe, M. Anomaly of Charge Transport of an Iodide/Tri-iodide Redox Couple in an Ionic Liquid and its Importance in Dye-sensitized solar cells, Chem. Commun. 2005, 2107 -2109.

14. Bai, Y.; Cao, Y.; Zhang, J.; Wang, M.; Li, R.; Wang, P.; Zakeeruddin, S. M.; Grätzel, M. Highperformance Dye-sensitized Solar Cells Based on Solvent-free Electrolytes Produced from Eutectic Melts, Nat. Mater. 2008, 7, 626-630. 
15. Papageorgiou, N.; Athanassov, Y.; Armand, M.; Bonhte, P.; Pettersson, H.; Azam, A.; Grätzel, M.; The Performance and Stability of Ambient Temperature Molten Salts for Solar Cell Applications, $J$. Electrochem. Soc. 1996, 143, $3099-3108$.

16. Zistler, M.; Wachter, P.; Wasserscheid, P.; Gerhard, D.; Hinsch, A.; Sastrawan, R.; Gores, H. J. Comparison of Electrochemical Methods for Triiodide Diffusion Coefficient Measurements and Observation of Non-Stokesian Diffusion Behaviour in Binary Mixtures of Two Ionic Liquids, Electrochim. Acta 2006, 52, 161- 169.

17. Thorsmølle, V.K.; Rothenberger, G.; Topgaard, D.; Brauer, J.C.; Kuang, D.-B.; Zakeeruddin, S.M.; Lindman, B.; Grätzel, M.; Moser, J.-E. Extraordinarily Efficient Conduction in a Redox-Active Ionic Liquid, ChemPhysChem 2011, 12, 145 - 149.

18. Grotthuss, C. J. T. Mémoire sur la Décomposition de l'Eau: et des Corps qu'elle Tient en Dissolution à l'Aide de l'Electricité Galvanique, Ann. Chim. 1806, 58, 54- 73.

19. Stegemann, H.; Reiche, A.; Schnittke, A.; Füllbier, H. Room-temperature Molten Polyiodides, Electrochim. Acta 1992, 37, 379- 383.

20. Svensson, P. H.; Kloo, L. Synthesis, Structure and Bonding in Polyiodide and Metal Iodide-Iodine Systems, Chem. Rev. 2003, 103, 1649- 1684.

21. Wachter, P.; Zistler, M.; Schreiner, C.; Fleischmann, M.; Gerhard, D.; Wasserscheid, P.; Barthel, J.; Gores, H.J. Temperature Dependence of the Non-Stokesian Charge Transport in Binary Blends of Ionic Liquids, J. Chem. Eng. Data 2009, 54, 491-497.

22. Bentley, C.L.; Bond, A.M.; Hollenkamp, A.F.; Mahon, P.J.; Zhang, J. Electrode Reaction and MassTransport Mechanisms Associated with the Iodide/Triiodide Couple in the Ionic Liquid 1-Ethyl-3methylimidazolium Bis(trifluoromethanesulfonyl)imide, J. Phys. Chem. C 2014, 118, 22439-22449.

23. Wang, Y.; Richards, W.D.; Ong, S.P.; Miara, L.J.; Kim, J. C.; Mo, Y.; Ceder, G. Design Principles for Solid-state Lithium Superionic Conductors, Nature Mat. 2015, 14, 1026-1031.

24. Nakakoshi, M. ; Shiro, M.; Fujimoto, T.; Machinami, T.; Seki, H.; Tashiro, M.; Nishikawa, K. Crystal Structure of 1-Butyl-3-methylimidazolium Iodide, Chem. Lett. 2006, 35(12), 1400-1401.

25. Canongia Lopes, J.N.; Deschamps, J.; Pádua, A.A.H. Modeling Ionic Liquids Using a Systematic All-Atom Force-Field J. Phys. Chem. B 2004, 108, 2038-2047.

26. Allen, M. P.; Tildesley, D. J. Computer Simulation of Liquids, Oxford University Press, 1987.

27. Hutter, J.; Iannuzzi, M.; Schiffmann, F.; VandeVondele, J. CP2K: Atomistic Simulations of Condensed Matter Systems. WIREs Comput Mol Sci, 2014, 4, 15-25. 
28. Perdew, J. P.; Burke, K.; Ernzerhof, M. Generalised Gradient Approximation Made Simple, Phys. Rev. Lett., 1996, 77, 3865 - 3868.

29. Grimme, S. Semiempirical GGA-type Density Functional Constructed with a Long-range Dispersion Correction. J. Comput. Chem., 2006, 27, 1787-1799.

30. Grimme, S.; et al., A Consistent and Accurate ab initio parameterisation of Density Functional Dispersion Correction (DFT-D) for the 94 Elements J. Chem. Phys., 2010, 132, 154104.

31. Kohanoff, J. ; Pinilla, C.; Youngs, T. G. A.; Artacho, E.; Soler, J. M. Dispersion Interactions in Room-temperature Ionic Liquids: Results from a Non-empirical Density Functional, J. Chem. Phys., 2011, $135,154505$.

32. Kim, K.S.; Shin, B.K.; Lee, H. Physical and Electrochemical Properties of 1-butyl-3methylimidazolium Bromide, 1-butyl-3-methylimidazolium Iodide, and 1-butyl-3methylimidazolium Tetrafluoroborate, Korean J. Chem. Eng. 2004, 21, 1010.

33. Jónsson, H.; Mills, G.; Jacobsen, K. W. in 'Classical and Quantum Dynamics in Condensed Phase Systems', 1998, ed. by B. J. Berne, G. Cicotti, and D. F. Coker, World Scientific.

34. Haynes, W.M. CRC Handbook of Chemistry and Physics, $95^{\text {th }}$ ed. 2014, Taylor \& Francis.

35. Hirshfeld, F.L. Bonded-Atom Fragments for Describing Molecular Charge Densities, Theoret. Chim. Acta 1977, 44, 129-138. 


\section{Figure list}

1. Schematic depiction of the $\mathrm{I}^{-} \cdots \mathrm{I}_{3}^{-}$moiety's (of length $\mathrm{d}_{41}$ ) bond-exchange process, with the incoming $\mathrm{I}^{-}$labelled as ' 4 ', with ' 1 ' as the departing one afterwards. Note that the approach of the $\mathrm{I}^{-}(4)$ need not be 'head-on', nor indeed the departure of 1 (i.e., collinear vis-à-vis the 2-3 vector): it is generally not so in the [BMIM][I] crystal phase.

2. Configurations (upper panels) and energies (bottom panels) along the pathways connecting six crystal states, in terms of a full cycle from, and back to, state 1. Energy set at zero for configuration ' $a$ ' on the left. The reaction coordinate for reorientations is the angular displacement of $\mathrm{I}_{3}^{-}, \alpha$, measured with respect to its initial orientation. For exchange processes the reaction coordinate is $\xi=d_{21}-d_{43}$, as defined in the text. Configurations: $\mathrm{a}=$ initial state for forward reaction; $\mathrm{b}=$ transition state; $\mathrm{c}=$ final state. Note there is naturally an angular $\mathrm{I}^{-}$approach/departure due to the symmetry of the [BMIM] crystal lattice. Full set of atomic coordinates provided as supplementary material.

3. Energies along the pathways for the three bond-exchange processes, in terms of the angle $\beta$ defined in the inset. $\mathrm{I}^{-}(4)$ is the approaching iodine, $\mathrm{I}^{-}(1)$ the leaving one. The configurations of the $\mathrm{I}^{-} \cdots \mathrm{I}_{3}^{-}$complex correspond to the transition state. The greater energy barrier of the first exchange process (evident in Fig. 2) results in a more 'twisted' bondexchange configuration (in relation to exchanges $2 \& 3$ ) and larger value of $\beta$ experienced along this pathway (almost $60^{\circ}$ at the TS).

4. Evolution of Hirshfeld charges (in $e$ units) on the iodine atoms during the reorientation and bond-exchange pathways. Note the 'mirror-image' symmetry for the outer atoms as they shift 'personality' upon the approach/departure of a (soon-to-become) iodide ion, as well as the parallel inversion symmetry between the entering and leaving (soon-to-become) iodide ions themselves. Atoms labels as in Fig. 2. 


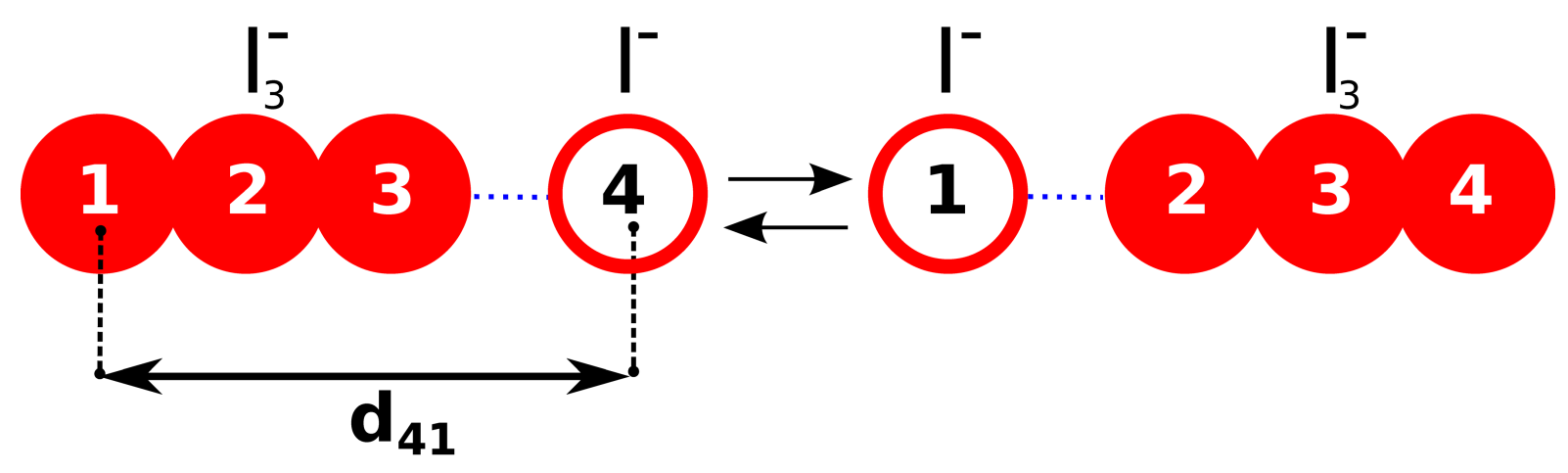

Fig. 1

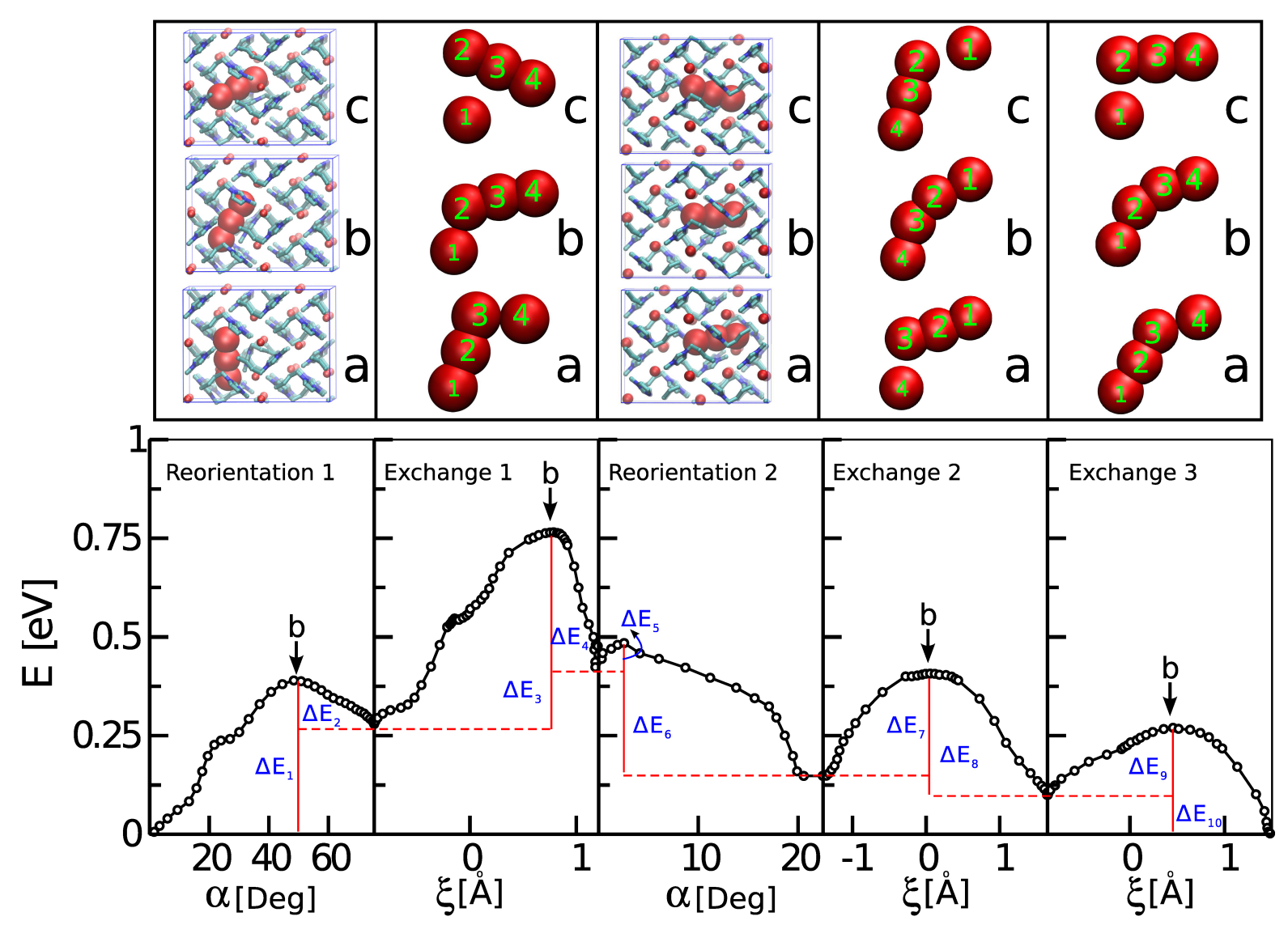

Fig. 2 


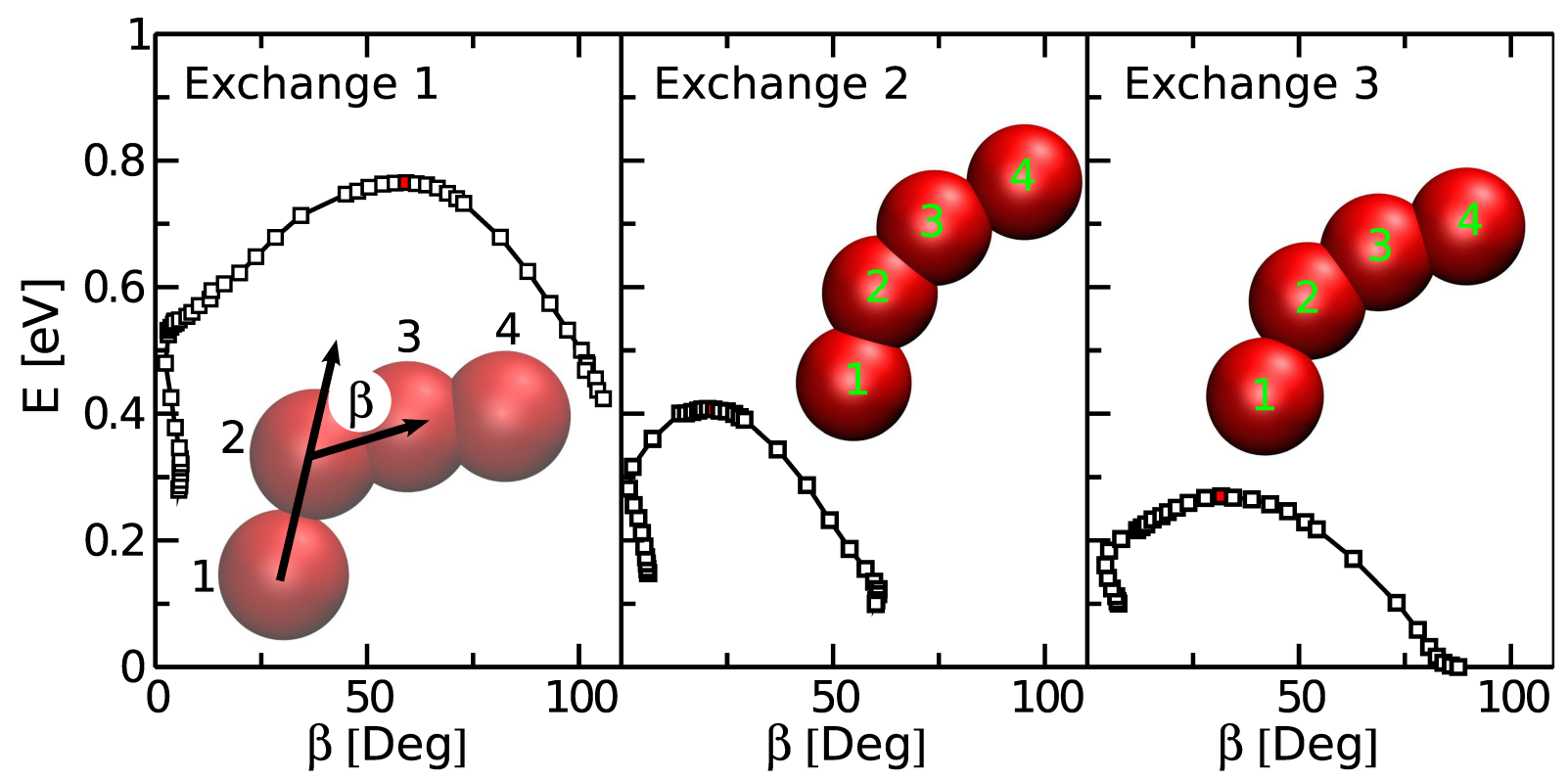

Fig. 3

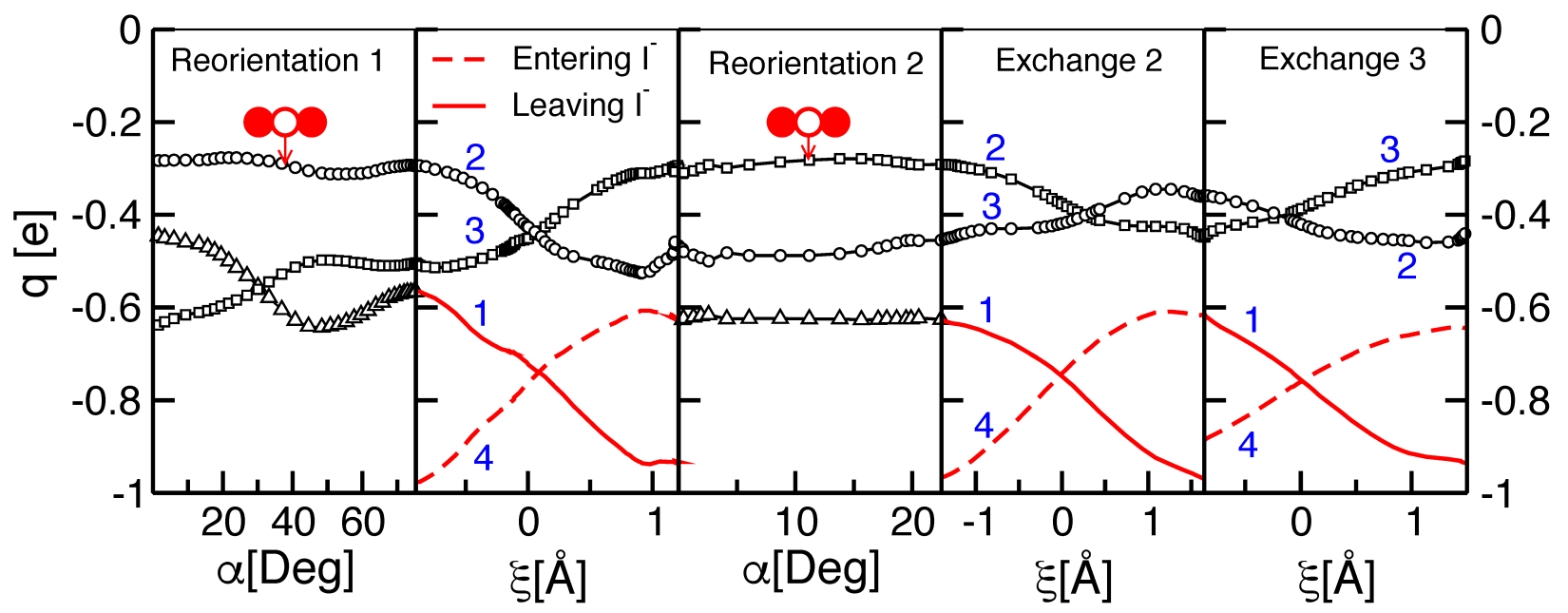

Fig. 4 


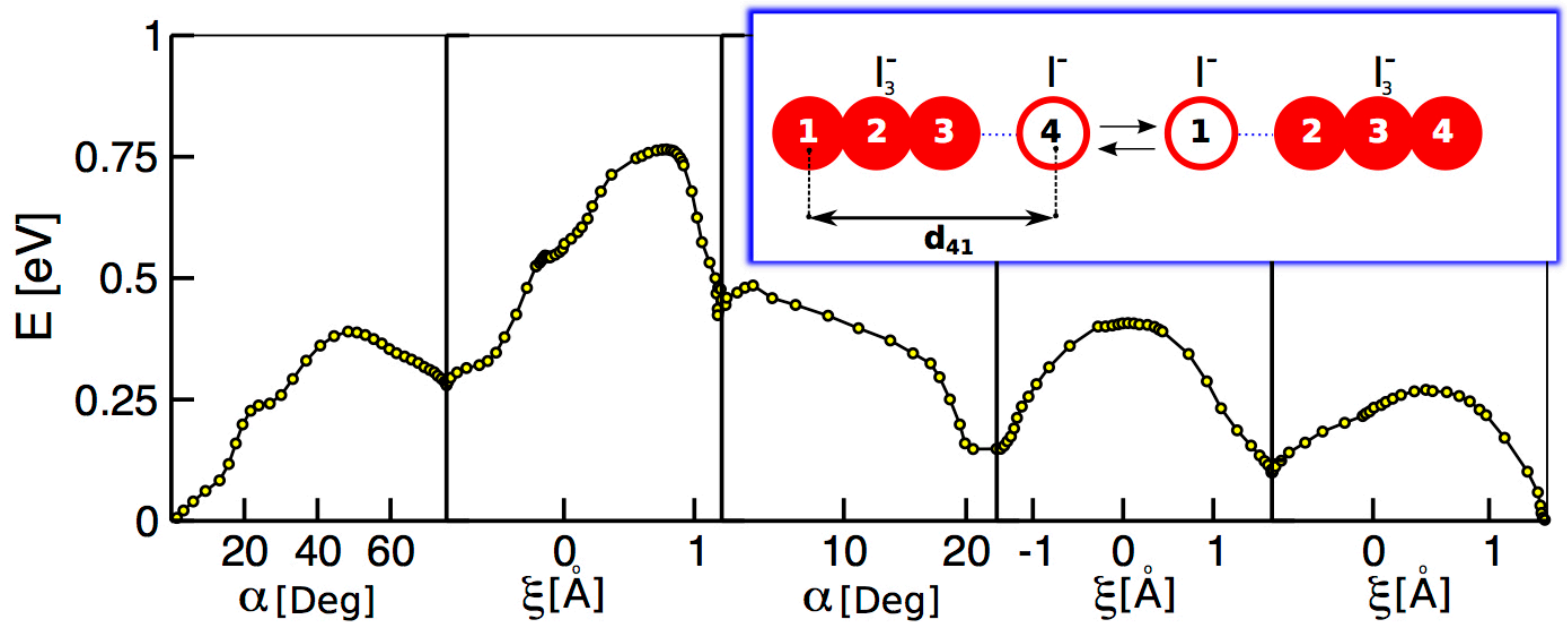

TOC Graphic 